\title{
Successful Management of Acquired Tracheomalacia of Patients With Amyotrophic Lateral Sclerosis: A Report of Three Cases
}

\author{
Jung Hyun Yang, MD, Tae Wan Kim, MD, Byeong Ju Lee, MD, Jin A Yoon, MD, \\ Myung Jun Shin, MD, PhD, Yong Beom Shin, MD, PhD \\ Department of Rehabilitation Medicine, Pusan National University Hospital, \\ Pusan National University School of Medicine, Busan, Korea
}

\begin{abstract}
Tracheomalacia is characterized by weakness of the tracheal walls and supporting cartilage. It results in dynamic compression of the airway, where the cross-sectional area of the trachea is reduced by expiratory compression. Acquired tracheomalacia results from complications associated with the use of endotracheal or tracheostomy tubes. In this report, we present three cases of patients with amyotrophic lateral sclerosis (ALS) successfully treated for tracheomalacia, including one case where the patient underwent surgery for combined tracheoesophageal fistula. We discuss the appropriate management strategies for tracheomalacia in patients with ALS. Through these case reports, we note the results of ALS patients who will have tracheostomy, and who are therefore at risk of sustaining a long term high cuff pressure, this study provides an evaluation for tracheomalacia and therapeutic management which should be considered for improving patient care outcomes.
\end{abstract}

Keywords Amyotrophic lateral sclerosis, Tracheomalacia, Tracheoesophageal fistula

\section{INTRODUCTION}

The incidence of acquired tracheomalacia in a patient can result from complications associated with the use of endotracheal or tracheostomy tubes. It is the case that with continued exposure to high cuff pressure, the cartilaginous tracheal rings soften, fragment, and then disap- pear, which in turn results in destruction of the supporting framework [1]. We report three cases of successfully managed tracheomalacia in patients with amyotrophic lateral sclerosis (ALS) who were using a tracheal tube.

Received May 19, 2017; Accepted August 7, 2017

Corresponding author: Jin A Yoon

Department of Rehabilitation Medicine, Pusan National University Hospital, Pusan National University School of Medicine, 179 Gudeok-ro, Seo-gu, Busan 49241, Korea. Tel: +82-51-240-7485, Fax: +82-51-247-7485, E-mail: yjk5289@naver.com

ORCID: Jung Hyun Yang (http://orcid.org/0000-0002-8480-2187); Tae Wan Kim (http://orcid.org/0000-0002-5418-9406); Byeong Ju Lee (http://orcid. org/0000-0002-7250-8909); Jin A Yoon (http://orcid.org/0000-0001-5762-0559); Myung Jun Shin (http://orcid.org/0000-0003-4010-0383); Yong Beom Shin (http://orcid.org/0000-0001-5026-1696).

(c) This is an open-access article distributed under the terms of the Creative Commons Attribution Non-Commercial License (http://creativecommons.org/ licenses/by-nc/4.0) which permits unrestricted noncommercial use, distribution, and reproduction in any medium, provided the original work is properly cited. Copyright ( 2018 by Korean Academy of Rehabilitation Medicine 


\section{CASE REPORTS}

\section{Case 1}

A 67-year-old male patient, who presented with dyspnea and unstable vital signs, was transferred to Pusan National University Hospital. He was diagnosed as having ALS 10 years ago. Subsequently, 3 years following diagnosis, he underwent a tracheostomy, with 24-hour ventilator assistance.

For several months prior to admission, the patient complained of continuous dyspnea and chest discomfort with leakage around his tracheostomy cuff, which resolved when the cuff was inflated with additional air by the caregiver. Upon admission, approximately 15-20 mL of air into the cuff measured by cuff manometer (VBM Medizintechnik, Sulz am Neckar, Germany) was required to achieve adequate sealing. The subsequent bronchoscopic examination and chest computed tomography (CT) revealed a dilated tracheal structure $(33.6 \mathrm{~mm} \times 37.5$ $\mathrm{mm})$, suggesting tracheomalacia with minor tracheoesophageal fistula (TEF) (Fig. 1).

At admission, the patient experienced acute oxygen desaturation (to 50\%) and a drop in blood pressure. The tube that was inserted at the time had the inner diameter

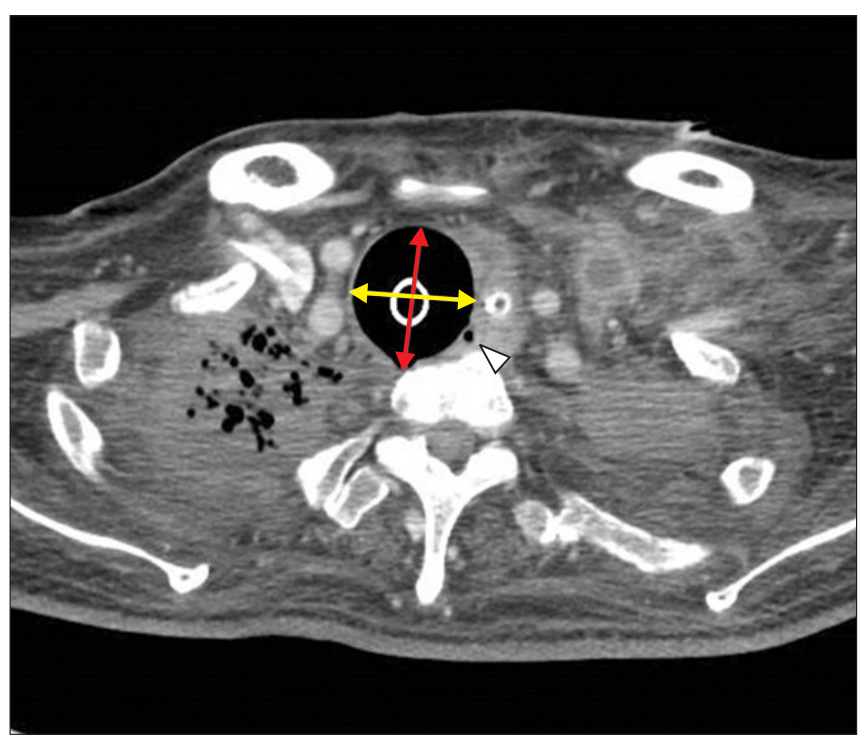

Fig. 1. Enlarged diameter of trachea (33.6 mm×37.5 mm) in inspiration computed tomography scan. A yellow arrow is a maximal transverse diameter $(33.6 \mathrm{~mm})$; red arrow, a maximal sagittal diameter $(37.5 \mathrm{~mm})$; white arrow head, tracheoesophageal fistula on tracheal level.
(ID) of $8.0 \mathrm{~mm}$, outer diameter (OD) of $11.9 \mathrm{~mm}$, and cuff pressure of $55 \mathrm{cmH}_{2} \mathrm{O}$ with $17 \mathrm{~mL}$ cuff inflation. The tracheostomy tube was immediately removed and replaced with an endotracheal tube, which resulted in improved oxygen saturation and airway pressure. The patient was taken to the intensive care unit (ICU) and we succeeded to replace ID of $8.0 \mathrm{~mm}$, OD of $11.0 \mathrm{~mm}$, and cuff resting diameter of $20 \mathrm{~mm}$ size of adjustable flange tracheostomy tube to maintain stable oxygen saturation after multiple tube changes. This particular tube is a longer than cuff tube length, and can be fixed on the desired depth by adjusting the fixing part of the neck. The cuff pressure of 30 $\mathrm{cmH}_{2} \mathrm{O}$ was maintained with $12 \mathrm{~mL}$ cuff inflation.

The tube was inflated with 10-15 mL of air, combined with a tracheal cuff pressure of less than $30 \mathrm{cmH}_{2} \mathrm{O}$, to minimize additional structural damage to the trachea. An additional bronchoscopy was performed to verify the location and size of the subtle fistula, and a 2 -cm defect was found at the left posterolateral wall of esophageal side. We carried out a sternothyroid muscular rotation flap surgery to close the tracheal defect.

After the operation, the patient continued with ventilator care in the general ward via an equivalent adjustable flange tracheal tube. The chest tube was removed, as the patient's improvement was confirmed by the use of chest radiographs. The patient was subsequently discharged from the hospital 78 days following admission.

\section{Case 2}

A 48-year-old male patient with ALS, presenting with several months of consistent air leakage at the tracheostomy site, visited Pusan National University Hospital. He was diagnosed as having ALS in 2005; he had undergone a tracheostomy 8 years previously and had received 24hour ventilator assistance. A 7.5-mm silicon tracheostomy tube had been used and, at the time of admission, nearly 15-20 mL of air with more than $90 \mathrm{cmH}_{2} \mathrm{O}$ of cuff pressure had been required to avoid air leakage.

A chest CT revealed a dilated trachea structure (26.97 $\mathrm{mm} \times 23.19 \mathrm{~mm})$, suggesting tracheomalacia around the cuff area. After multiple tube changes, we used an ID of $7.5 \mathrm{~mm}$, OD of $10.4 \mathrm{~mm}$, and cuff resting diameter of 31.0 $\mathrm{mm}$ double cuff tracheostomy tube. Unlike a previous cuffed tracheostomy tube, the double cuff tube can use the alternation of the cuff inflation to minimize risk of injury on high risk tracheal mucosa. 
At review, there was nearly 6-8 $\mathrm{mL}$ of distal balloon inflation, with a cuff pressure measuring less than 25 $\mathrm{cmH}_{2} \mathrm{O}$, was recorded and measured, minimizing additional structural damage to the trachea. The patient was discharged from the hospital 5 days following admission.

\section{Case 3}

A 71-year-old male patient who presented with redness and discharge around the tracheostomy site was hospitalized. Upon review of his condition, consistent air leakage around the tracheal cuff was also noted. He was diagnosed as having ALS 12 years ago; he had a tracheostomy, with 24-hours of ventilator assistance, 4 years after diagnosis. Nearly $10 \mathrm{~mL}$ of air was required to achieve an adequate sealing effect, with $80 \mathrm{cmH}_{2} \mathrm{O}$ of tracheal cuff pressure. A chest CT of the patient revealed a disrupted trachea structure and dilated trachea $(28.66 \mathrm{~mm} \times 24.39$ $\mathrm{mm}$ ), suggesting tracheomalacia with abscess formation and an increased soft tissue density at the posterior aspect of the tracheostomy site (Fig. 2).

We started antibiotic therapy for the abscess, and replaced an ID of $7.0 \mathrm{~mm}$, OD of $9.6 \mathrm{~mm}$, and cuff resting diameter of $24 \mathrm{~mm}$ size of adjustable flange tracheostomy

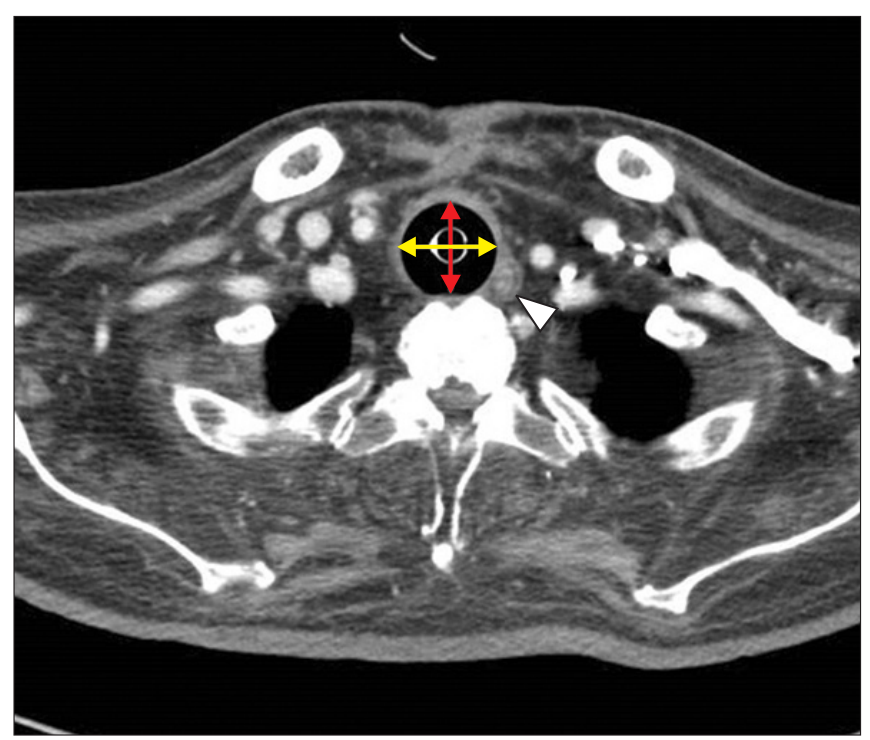

Fig. 2. Enlarged diameter of the trachea $(28.66 \mathrm{~mm} \times 24.39$ $\mathrm{mm}$ ) with abscess formation and increase in soft tissue density at the posterior aspect of tracheostomy site in computed tomography view. A yellow arrow is a maximal transverse diameter $(28.66 \mathrm{~mm})$; red arrow, a maximal sagittal diameter $(24.39 \mathrm{~mm})$; white arrow head, abscess on tracheal level. tube to avoid air leakage. After multiple tracheal ballooning trials, we setup $7 \mathrm{~mL}$ of air combined with a tracheal cuff pressure of around $40 \mathrm{cmH}_{2} \mathrm{O}$ without air leakage. The patient was subsequently discharged from the hospital 14 days following admission.

\section{DISCUSSION}

Tracheomalacia is characterized by weakness of the tracheal walls and supporting cartilage. It is defined as dynamic compression of the airway, where the crosssectional area of the trachea is reduced by expiratory compression [2]. The incidence of acquired tracheomalacia is caused by any process that affects the change in the compliance of the trachea, and disrupts the uniformity of caliber change of the trachea [3]. The normal transverse diameter of the trachea ranges between 15 and $25 \mathrm{~mm}$ in men, and 10 to $21 \mathrm{~mm}$ in women, but in this study we noted that the tracheal diameter in all three patients was dilated $[4,5]$. As described in our first case, the most frequent cause of acquired tracheomalacia is an indwelling tracheostomy and endotracheal tube with an inflatable cuff $[6,7]$ and some of the cases develop into TEF at the level of the cuff. Recently however, the current recommendations include the utilization of a cuff pressure range between 20 and $30 \mathrm{cmH}_{2} \mathrm{O}$ in adult patients to prevent tracheal injury [8]. We have noted that the overinflation of the cuff causes tracheomalacia, TEF, and tracheal rupture due to tracheal mucosal blood flow injury. In the opposite case, the noted under-inflation of the cuff can occur air leakage, inadequate ventilation, microaspiration and pneumonia. In the case of tracheomalacia as in the previous case, it is very important that the position of the cuff is repositioned in that case, so as not to give mucosal injury at the same position.

After placing an endotracheal tube to improve oxygen saturation in our first case, we tried multiple tube changes, and eventually used it as an adjustable flange tube. For two of our patients, the early diagnosis of tracheomalacia and tube changes subsequently served the patient, and avoided consistent air leakage, and also reduced the patient's hospitalization period. Therefore, it is important to reposition the cuff level by choosing the right types of tube, in order to avoid the mucosal injury to the patient. After changing the tube and reducing the cuff pressure, there was no air leakage or microaspiration, 
and the ventilator modes were not changed in all three cases. However, intermittent air leak around the cuff was observed after home discharge, which improved when the tracheostomy tube was correctly positioned.

In order to manage the tracheostomy tube properly, the tracheostomy cuff pressure and air leakage should be measured periodically. If there is a problem in any of these apparatus, the tube should be changed to another type, such as adjustable flange or double cuff tube, instead of the subsequent use of a cuff inflation. We hope that this presented information will encourage more medical staff, including rehabilitation specialists, to adequately manage the use of a tracheostomy cuff to prevent the incidence of tracheomalacia, especially in patients diagnosed with ALS.

\section{CONFLICT OF INTEREST}

No potential conflict of interest relevant to this article was reported.

\section{ACKNOWLEDGMENTS}

This work was supported by clinical research grant from Pusan National University Hospital in 2017.

\section{REFERENCES}

1. Reed MF, Mathisen DJ. Tracheoesophageal fistula. Chest Surg Clin N Am 2003;13:271-89.

2. Jokinen K, Palva T, Sutinen S, Nuutinen J. Acquired tracheobronchomalacia. Ann Clin Res 1977;9:52-7.

3. Feist JH, Johnson TH, Wilson RJ. Acquired tracheomalacia: etiology and differential diagnosis. Chest 1975; 68:340-5.

4. Epstein PE. Radiologic diagnosis of diseases of the chest. Ann Intern Med 2001;135:1097.

5. Breatnach E, Abbott GC, Fraser RG. Dimensions of the normal human trachea. AJR Am J Roentgenol 1984; 142:903-6.

6. Flege JB. Tracheoesophageal fistula caused by cuffed tracheostomy tube. Ann Surg 1967;166:153-6.

7. Pearson FG, Andrews MJ. Detection and management of tracheal stenosis following cuffed tube tracheostomy. Ann Thorac Surg 1971;12:359-74.

8. Hockey CA, van Zundert AA, Paratz JD. Does objective measurement of tracheal tube cuff pressures minimise adverse effects and maintain accurate cuff pressures? A systematic review and meta-analysis. Anaesth Intensive Care 2016;44:560-70. 